HWM-09-5

EMPG-09-10

\title{
Quantum Gravity, Field Theory and Signatures of Noncommutative Spacetime ${ }^{1}$
}

\author{
Richard J. Szabo \\ Department of Mathematics \\ Heriot-Watt University \\ Colin Maclaurin Building, Riccarton, Edinburgh EH14 4AS, U.K. \\ and Maxwell Institute for Mathematical Sciences, Edinburgh, U.K. \\ Email: R.J.Szabo@ma.hw.ac.uk
}

\begin{abstract}
A pedagogical introduction to some of the main ideas and results of field theories on quantized spacetimes is presented, with emphasis on what such field theories may teach us about the problem of quantizing gravity. We examine to what extent noncommutative gauge theories may be regarded as gauge theories of gravity. UV/IR mixing is explained in detail and we describe its relations to renormalization, to gravitational dynamics, and to deformed dispersion relations in models of quantum spacetime of interest in string theory and in doubly special relativity. We also discuss some potential experimental probes of spacetime noncommutativity.
\end{abstract}

\footnotetext{
${ }^{1}$ Based on Plenary Lecture delivered at the XXIX Encontro Nacional de Física de Partículas e Campos, São Lourenço, Brasil, September 22-26, 2008.
} 


\section{Contents}

1 Introduction $\quad 1$

2 Spacetime quantization $\quad 3$

2.1 Snyder's spacetime . . . . . . . . . . . . . . . . . . . 3

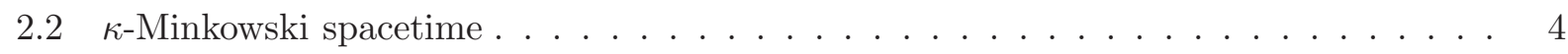

2.3 Three-dimensional quantum gravity . . . . . . . . . . . . . . . 5

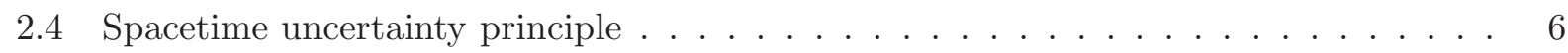

2.5 Physics in strong magnetic fields . . . . . . . . . . . . . . . 7

2.6 Noncommutative geometry in string theory . . . . . . . . . . . . 8

3 Field theory on quantized spacetimes $r$

3.1 Formalism . . . . . . . . . . . . . . . . . . . . . . 9

$3.2 \mathrm{UV} / \mathrm{IR}$ mixing $\ldots \ldots \ldots \ldots \ldots \ldots \ldots \ldots \ldots \ldots$

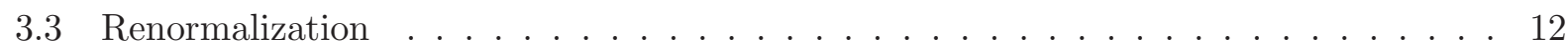

4 Noncommutative gauge theory of gravity $\quad 14$

4.1 Gauge interactions . . . . . . . . . . . . . . . . . . . . . . 14

4.2 Gravity in noncommutative gauge theories . . . . . . . . . . . . . . . . 15

4.3 Spacetime disappears . . . . . . . . . . . . . . . . . . . . . . 15

4.4 Gravitational dynamics and UV/IR mixing . . . . . . . . . . . . . . . . 17

5 Signatures of spacetime noncommutativity $r$

5.1 Violations of Lorentz invariance and causality . . . . . . . . . . . . . . . 18

5.2 Dispersion relations and UV/IR mixing . . . . . . . . . . . . . . . 19

\section{Introduction}

The validity of certain models or hypotheses in theoretical physics is sometimes argued on the basis of length scales. In modern theoretical physics, physical phenomena occur from down at the fundamental Planck scale

$$
\ell_{\mathrm{P}}=\sqrt{\hbar G / c^{3}} \simeq 1.6 \times 10^{-33} \mathrm{~cm}
$$

all the way up to the radius of the observable universe

$$
\ell_{\text {universe }} \simeq 4.4 \times 10^{24} \mathrm{~cm} \simeq\left(2.7 \times 10^{61}\right) \ell_{\mathrm{P}} .
$$

We know that quantum field theory works well in describing the pertinent physics at least down to the LHC scale

$$
\ell_{\mathrm{LHC}} \simeq 2 \times 10^{-18} \mathrm{~cm}
$$


What happens below this scale, but perhaps still in the regime above the Planck scale, is a question that will not be answered satisfactorily by experiment for many years to come. In the passing time, theoretical physicists may reap the pastures of this unknown regime and speculate on the mathematical foundations that the correct modification of quantum field theory might assume at such length scales.

Such is the speculative nature of the notion of "spacetime quantization". In this somewhat radical scenario, the local coordinates $x^{\mu}$ are promoted to hermitean operators satisfying spacetime noncommutativity commutator relations

$$
\left[x^{\mu}, x^{\nu}\right]=\mathrm{i} \theta^{\mu \nu}
$$

where $\theta^{\mu \nu}$ is a real antisymmetric matrix of dimension (length) ${ }^{2}$. If this matrix is constant, then the commutators (1.4) generate a Heisenberg algebra and imply spacetime uncertainty relations

$$
\Delta x^{\mu} \Delta x^{\nu} \geq \frac{1}{2}\left|\theta^{\mu \nu}\right|
$$

This is derived in the same standard way that one would derive Heisenberg's uncertainty principle from canonical commutation relations between coordinates $x^{\mu}$ and momenta $p_{\nu}$ in quantum mechanics. In this sense the dimensionful noncommutativity parameters $\theta^{\mu \nu}$ are analogous to Planck's constant $\hbar$ in the phase space quantization relations

$$
\left[x^{\mu}, p_{\nu}\right]=\mathrm{i} \hbar \delta_{\nu}^{\mu}
$$

In a quantum phase space, points no longer exist and are replaced with Planck cells of size $\hbar$. von Neumann thus dubbed the study of geometrical properties of quantum mechanics as "pointless" geometry. In modern parlance of quantized spacetime, this branch of mathematics has come to be known as "noncommutative geometry". The points of a quantized spacetime become "fuzzy" and are replaced with cells whose size is set by the noncommutativity length scale $\ell \simeq \sqrt{\theta}$. In general, the tensor $\theta^{\mu \nu}$ can depend on the spacetime coordinates, and even on the momenta (in which case the algebra of canonical commutation relations is replaced with an algebra of pseudo-differential operators). We shall see some explicit examples in what follows.

The proposal of spacetime quantization arises in two major problems of modern high-energy physics. The first is the problem of renormalization. Just as the Heisenberg uncertainty principle enables one to avoid the ultraviolet catastrophe in quantum mechanics, the replacement of points with spacetime cells is a means in which to tame the ultraviolet divergences of quantum field theory, as an elegant symmetry preserving alternative to lattice or cutoff regularizations. This turns out to be an extremely subtle issue and will be discussed in detail in the following. The second is the problem of quantum gravity. It has been long suspected that classical general relativity breaks down at the Planck scale $\ell_{\mathrm{P}}$, where quantum gravitational effects become important. In particular, the classical Riemannian geometry of spacetime must be replaced by some other mathematical framework. Since, according to Einstein's theory, gravity affects spacetime geometry, quantum gravity should quantize spacetime. The precise manner in which this quantization occurs is of course not entirely understood and is one of the biggest challenges facing modern theoretical physics. Noncommutative geometry may in this way provide at least some guidance as to how to handle spacetime structure at very short distances.

In what follows we shall argue that these two fundamental problems are in fact related to each other within a systematic and unified framework of field theory on quantized spacetimes. This theoretical framework is called noncommutative field theory and it may be a relevant physical model at scales in between $\ell_{\mathrm{P}}$ and $\ell_{\mathrm{LHC}}$. In fact, one of the main threads of research in this field has been related to studies of energetic cosmic rays, as we will discuss further below. In the following we will 
study this relationship in some detail. These field theories provide fruitful avenues of exploration for several reasons, that will be explained in more depth below. Firstly, some quantum field theories are better behaved on noncommutative spacetime than on ordinary spacetime. In fact, some are completely finite, even non-perturbatively. In this manner spacetime noncommutativity presents an alternative to supersymmetry or string theory. Secondly, it is a useful arena for studying physics beyond the standard model, and also for standard physics in strong external fields. Thirdly, it sheds light on alternative lines of attack to addressing various foundational issues in quantum field theory, for instance the renormalization and axiomatic programmes. Finally, it naturally relates field theory to gravity. Since the field theory may be easier to quantize, this may provide significant insights into the problem of quantizing gravity.

This survey is intended for high-energy physicists who are non-specialists in noncommutative field theory. As such, technical details are kept to a minimum and we refer to various other literature throughout for the relevant formalisms. Foundational aspects of noncommutative quantum field theory are treated in $[1,2]$. Brief reviews of the relationship between spacetime noncommutativity and quantum gravity may be found in $[3,4]$. A detailed overview of the renormalization programme for noncommutative field theories is given in [5]. The connection between spacetime noncommutativity and experimental signatures of Lorentz violation are treated in [6]. The restoration of Lorentz symmetry is related to the problem of renormalization of noncommutative field theories in [7]. The interplay between noncommutative field theory and physics in strong magnetic fields is discussed in [8]. There are many other topics in this vast field that are not touched upon in this review. Furthermore, the bibliography is not meant to be exhaustive, and we apologise in advance to those concerned for the omissions.

\section{Spacetime quantization}

In this section we will provide a (non-exhaustive) list of contexts in which noncommutative spacetimes play a prominent role, particularly those which can be argued to arise in various approaches to quantum gravity, as well as the applications mentioned in $\S 1$. The aim is to set up some explicit models where noncommutative geometry is naturally interlaced with the problem of quantizing gravity, at least at a kinematical level. Later on we shall examine this relationship from a more dynamical perspective.

\subsection{Snyder's spacetime}

The idea of spacetime noncommutativity is in fact very old. It is usually attributed to Heisenberg who proposed it in the late 1930's as a means of regulating the ultraviolet divergences which plague quantum field theory. Heisenberg suggested this idea in a letter to Peierls, who actually applied it in a non-relativistic context of electronic systems in external magnetic fields (we will come back to this application later on). Peierls told Pauli about the idea, who then told Oppenheimer. Oppenheimer gave the problem to his graduate student Snyder, whose is acredited today with the original paper on noncommutative spacetime [9].

In a suitable basis, the algebra underlying Snyder's spacetime may be presented as a modification of the phase space canonical commutation relations given by

$$
\begin{aligned}
& {\left[x^{\mu}, x^{\nu}\right]=\mathrm{i} \ell^{2} \hbar^{-1}\left(x^{\mu} p^{\nu}-x^{\nu} p^{\mu}\right),} \\
& {\left[x^{\mu}, p_{\nu}\right]=\mathrm{i} \hbar \delta^{\mu}{ }_{\nu}+\mathrm{i} \ell^{2} \hbar^{-1} p^{\mu} p_{\nu},} \\
& {\left[p_{\mu}, p_{\nu}\right]=0 .}
\end{aligned}
$$


This algebra involves a fundamental minimal length $\ell$, the scale of noncommutativity as in $\S 1$, such that the "classical" phase space of quantum mechanics is recovered at $\ell=0$. The original motivation behind these relations was that the introduction of the length scale $\ell$ is tantamount to regarding hadrons in quantum field theory as extended objects, because at the time renormalization theory was regarded as "a distasteful procedure" [9]. The commutation relations (2.1) describe a discrete spacetime which is compatible with Lorentz invariance.

There is a natural way to derive the algebra (2.1) in 3+1-dimensions in terms of a dimensional reduction from $4+1$-dimensions as the angular momentum generators of the higher-dimensional Lorentz group $S O(1,4)$ [10] (which clarifies at least the first relation in $(2.1)$ ). In this way the generators of Snyder's spacetime are naturally interpreted as the generators which preserve a fourdimensional de Sitter space inside five-dimensional Minkowski space, and are naturally invariant under both five-dimensional and four-dimensional Lorentz transformations. This reduction is consistent with the fact that the phase space in this model contains a curved momentum space, as signified by the momentum dependence on the right-hand side of the commutation relations (2.1).

The death of Snyder's spacetime, within the context in which it was proposed at the time, was triggered by the eventual success of the renormalization programme in quantum field theory. The historic overwhelmingly successful agreement with experiment of the calculations of both the Lamb shift in hydrogen and the anomalous magnetic moment of the electron from radiative corrections in quantum electrodynamics put the more conventional regularization techniques at the forefront of modern quantum field theory. However, this spacetime emerged again more than half a century later in the somewhat unexpected and surprising context of quantum gravity.

\section{2 $\kappa$-Minkowski spacetime}

The phase space commutation relations of the $\kappa$-Minkowski spacetime can be written in the bicrossproduct basis as $[11,12]$

$$
\begin{aligned}
{\left[x^{\mu}, x^{\nu}\right] } & =\frac{\mathrm{i}}{\kappa}\left(x^{\mu} \xi^{\nu}-x^{\nu} \xi^{\mu}\right), \\
{\left[x^{\mu}, p_{\nu}\right] } & =\mathrm{i} \hbar \delta^{\mu}{ }_{\nu}+\frac{\mathrm{i}}{\kappa}\left(p^{\mu} \xi_{\nu}+p_{\nu} \xi^{\mu}\right), \\
{\left[p_{\mu}, p_{\nu}\right] } & =0
\end{aligned}
$$

where the lightlike four-vector $\xi^{\mu}$ has components $\xi^{0}=1, \xi^{i}=0$, and $\kappa$ is a mass scale. In the notation of $\S 2.1$, we identify $\kappa \simeq \hbar / \ell$ as a very large energy. In a somewhat less covariant formulation than in (2.2), only commutators involving the time components $x^{0}=c t$ and $p_{0}=E / c$ are deformed by the mass scale in (2.2). In fact, this spacetime is equivalent to Snyder's spacetime [13] through a mapping between the generators of the algebras (2.1) and (2.2). This may seem surprising, given that the Snyder coordinates are determined by Lorentz transformations preserving fourdimensional de Sitter space, rather than Minkowski space, but the mapping is determined by highly non-local transformations involving, for example, momentum-dependent redefinitions of the spacetime coordinates.

The $\kappa$-Minkowski algebra has been of interest lately as the noncommutative spacetime underlying certain realizations of doubly special relativity [14]. These modifications of special relativity contain, in addition to the speed of light $c$, a second, short-distance observer-independent length scale, usually identified as the Planck scale $\ell=\ell_{\mathrm{P}}$. They have been proposed as a consistent description of quantum gravity in flat spacetime, which are thereby amenable to phenomenological tests. In these models the effective spacetime metric, or alternatively the Poincare symmetry of flat Minkowski spacetime, is deformed by energy according to the relations (2.2), reflecting again the 
curvature of momentum space. See $[15,16]$ for an explicit canonical framework which reflects the deformed symmetries of doubly special relativity as a non-linear realization of the Lorentz group.

This noncommutative deformation of Minkowski space modifies the dispersion relations to [17]

$$
E^{2}=p^{2} c^{2}-m^{2} c^{4}\left(1-\frac{\xi \cdot p}{\hbar \kappa}\right)^{2}
$$

Thus the speed of a photon in $\kappa$-Minkowski spacetime depends on its energy $E$. This is consistent because the dependence is through the small dimensionless combination $\ell_{\mathrm{P}} E$. These predictions have been tested with some success against measurements of astrophysical gamma-ray bursts as measured, for example, by the GLAST and MAGIC telescopes [6]. In the low-energy limit $\kappa \rightarrow \infty$, whereby the algebra (2.2) reduces to the usual phase space commutation relations of ordinary Minkowski space, these results are all in agreement with the macroscopic predictions of classical general relativity.

\subsection{Three-dimensional quantum gravity}

Perhaps the most precise dynamical realization of the connections between noncommutative field theory and quantum gravity alluded to in $\S 2.2$ comes from a non-perturbative model of quantum gravity in three spacetime dimensions [18]. One starts with a spin foam model of three-dimensional gravity coupled to spinless matter, and then integrates out the gravitational degrees of freedom in the path integral to produce an effective action for the scalar fields. In this way one finds that the effective interactions can be encoded in a scalar field theory on the $S O(1,2)$ Lie algebra noncommutative spacetime

$$
\begin{aligned}
& {\left[x^{\mu}, x^{\nu}\right]=\mathrm{i} \ell \epsilon^{\mu \nu \lambda} x_{\lambda},} \\
& {\left[x^{\mu}, p_{\nu}\right]=\mathrm{i} \sqrt{\hbar^{2}-\ell^{2} p^{2}} \delta^{\mu}{ }_{\nu}-\mathrm{i} \ell \epsilon^{\mu \lambda}{ }_{\nu \lambda} p^{\lambda},} \\
& {\left[p^{\mu}, p^{\nu}\right]=0 .}
\end{aligned}
$$

The three-dimensional spacetime algebra (2.4) also arises in the context of Lorentz covariant spacetime uncertainly relations [19] (such relations are discussed in more detail below). This spacetime again has a $\kappa$-deformed Poincaré symmetry, together with modified dispersion relations

$$
E^{2}=\boldsymbol{p}^{2} c^{2}-\left(\frac{\sinh \left(\ell \hbar^{-1} m c^{2}\right)}{\ell \hbar^{-1}}\right)^{2}
$$

This dispersion relation matches (2.3) at leading orders in the limit $\ell \rightarrow 0$.

Thus in this case, doubly special relativity arises in the low-energy limit of quantum gravity. The mechanism behind this emergence is an effective noncommutative quantum field theory. The properties of this noncommutative field theory and its precise relationship to $2+1$-dimensional gravity is currently under active investigation. For example, certain properties of the (curved) momentum space associated to (2.4) indicate that some modifications seem necessary. The periodicity of the momentum space ruins unitarity of the noncommutative field theory, while the absence of arbitrary negative energy means that it cannot correspond to the momentum space of massive particles coupled to $2+1$-dimensional gravity. These problems are analysed in [20], where it is proposed that an extension of the momentum space of the noncommutative field theory to its universal covering group may resolve these problems. 


\subsection{Spacetime uncertainty principle}

Despite their appeal as direct relatives of quantum gravity and high-energy astrophysical processes, the models of noncommutative spacetime we have thus far considered are far too complex to be useful for detailed direct study of the dynamics of quantum fields defined on them. They are best handled using Hopf algebraic techniques and methods of braided quantum field theory [21, 22]. We seek a somewhat simpler setting which is amenable to the standard perturbative methods of quantum field theory, but at the same time still captures the nature of Planck scale physics. We will then be able to study to what extent the field theoretic model captures the dynamics of gravitational interactions. The models which we will discuss in detail in the remainder of this survey can be motivated within the above context by the simple semi-classical argument of [23]. This argument is based on combining only fundamental postulates of general relativity with quantum mechanics, and it demonstrates that spacetime quantization, and more generally noncommutative geometry, is expected to be a generic feature of any theory of quantum gravity, consistent with what we observed in $\S 2.3$.

Suppose that we try to probe physics at the Planck scale $\ell_{\mathrm{P}}$. Then the Compton wavelength of any probe must be smaller than $\ell_{\mathrm{P}}$. But this means that there is a huge mass $m \geq \hbar / \ell_{\mathrm{P}} c$ concentrated in a tiny volume $\ell_{\mathrm{P}}^{3}$, and the energy density is large enough that it forms a black hole. The event horizon has size determined by the Schwarzschild radius which is of order $m$, and it thereby hides the measurement we set out to make as no information can escape from the interior of the black hole through its horizon. To avoid this problem, there must be a mechanism in place which prevents the occurence of such a gravitational collapse.

The mechanism proposed by [23] is to introduce a fundamental length $\ell$, that we may wish to identify with the Planck scale $\ell=\ell_{\mathrm{P}}$, which limits both spatial and temporal localization via the spacetime uncertainty relations

$$
\Delta x^{0} \sum_{i} \Delta x^{i} \geq \ell_{\mathrm{P}}^{2} \quad \text { and } \quad \sum_{i<j} \Delta x^{i} \Delta x^{j} \geq \ell_{\mathrm{P}}^{2} .
$$

Then, if we wish to make a measurement along, say, the $j$-th direction of space, the energy of our probe will spread out over a perpendicularly directed disk of radius $\Delta x^{i}$, such that the induced gravitational potential vanishes as we try to localize the space coordinate $x^{j}$, i.e. in the limit where $\Delta x^{i} \rightarrow \infty$. A similar reasoning applies to localization of the time coordinate $x^{0}$. As indicated in $\S 1$, a concrete model of quantum spacetime which fulfills these requirements, and which is good for studying quantum field theory thereon, has the quantum phase space commutation relations

$$
\begin{aligned}
& {\left[x^{\mu}, x^{\nu}\right]=\mathrm{i} \theta^{\mu \nu}=\text { constant }} \\
& {\left[x^{\mu}, p_{\nu}\right]=\mathrm{i} \hbar \delta^{\mu}{ }_{\nu},} \\
& {\left[p_{\mu}, p_{\nu}\right]=0}
\end{aligned}
$$

with

$$
\theta_{\mu \nu} \theta^{\mu \nu}=0 \quad \text { and } \quad \epsilon_{\mu \nu \lambda \rho} \theta^{\mu \nu} \theta^{\lambda \rho}=-8 \ell_{\mathrm{P}}^{4} .
$$

One of the main simplifications arising in this class of spacetimes is that their momentum spaces are flat, in contrast to those considered earlier in this section. Only the commutators between two spacetime coordinates are deformed.

In the original treatment of [23], the constant noncommutativity parameters $\theta^{\mu \nu}$ are treated as "dynamical" variables, in the sense that their constant values are allowed to vary subject to the 
constraints (2.8). This means that the algebra of spacetime coordinates $x^{\mu}$ is enlarged to include $\theta^{\mu \nu}$ on an equal footing as central elements, so that

$$
\left[x^{\mu}, \theta^{\nu \lambda}\right]=0 \text {. }
$$

In this enlarged algebra, the action of a Lorentz transformation on $x^{\mu}$ can be compensated in the commutation relations (2.7) by letting $\theta^{\mu \nu}$ transform as a covariant tensor of rank two under $S O(1,3)$. In this way, the quantum spacetime is compatible with Lorentz invariance. However, in the following we will instead think of $\theta^{\mu \nu}$ as a fixed and arbitrary background tensor field, at the cost of breaking Lorentz symmetry and the explicit uncertainty relations (2.6). Such a Lorentz violation could have experimental signatures, as we will discuss in $\S 5$. The motivation behind this restriction comes from various physical contexts in which systems are subjected to strong external background fields, some examples of which are discussed below. It also simplifies somewhat the analysis of the resulting noncommutative quantum field theories.

\subsection{Physics in strong magnetic fields}

The simplest prototypical example whose dynamics induces a quantum space is the Landau problem. The Landau problem considers the quantum mechanics of a non-relativistic system of electrons

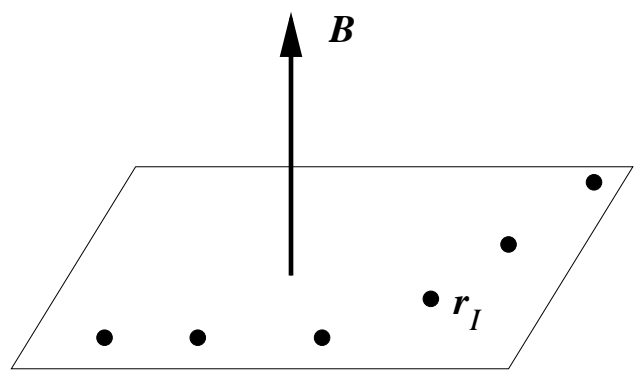

confined to a plane $\boldsymbol{x}=(x, y, 0)$ and subjected to a uniform background magnetic field of strength $B$ which is applied in the direction perpendicular to the plane of motion. The lagrangian for each electron is given by

$$
\mathcal{L}_{m}=\frac{m}{2} \dot{\boldsymbol{x}}^{2}-\frac{e}{c} \dot{\boldsymbol{x}} \cdot \boldsymbol{A},
$$

where

$$
A_{x}=-\frac{B}{2} y \quad \text { and } \quad A_{y}=\frac{B}{2} x
$$

are the non-zero components of the corresponding vector potential in the symmetric gauge.

The quantization of this system is an elementary exercise in senior undergraduate level quantum mechanics. One can map the Hamiltonian of this model onto that of a harmonic oscillator, whose spectrum yields the Landau levels. The lowest Landau level is the ground state of this harmonic oscillator. In the strong field limit $e B \gg m$, the spacing between Landau levels becomes infinite and the spectrum projects onto the lowest Landau level. The lagrangian (2.10) in this regime truncates to

$$
\mathcal{L}_{0}=-\frac{e B}{2 c}(\dot{x} y-\dot{y} x) .
$$

This reduced Lagrangian is of first order in time derivatives. The phase space therefore becomes degenerate and collapses onto the configuration space. Thus canonical quantization gives a noncommutative space having the commutators

$$
[x, y]=\mathrm{i} \theta \quad \text { with } \quad \theta=\frac{\hbar c}{e B} .
$$


This observation can have many applications to systems which are subjected to strong magnetic fields. As mentioned in $\S 1$, the first application was the famous Peierls substitution [24]. This utilizes the Schrödinger representation of the Heisenberg commutation relations (2.13) to compute the first order energy shift due to an impurity potential $V(x, y)$ in perturbation theory of the lowest Landau level of the electronic system. More recently, based on the analogy between the canonical quantization of the Landau problem above and that of Chern-Simons gauge theory [25], it has been used to motivate a noncommutative Chern-Simons theory formulation of the fractional quantum Hall effect which has been shown to provide a much better microscopic description of the dynamics than the commutative effective field theory [26]-[28]. It may also be useful for studying certain models of charged polymer growth in magnetic fields in terms of random walks with self-avoiding interaction [29], and it may even find applications to the description of bound states in non-abelian gauge theories, in particular to quark confinement [30]. See $[5,8]$ for further discussion of these applications.

\subsection{Noncommutative geometry in string theory}

Our final example is the context in which noncommutative spaces arise in string theory, which has been mostly responsible for the huge surge of activity in noncommutative field theory over the past decade. Strings naturally come with a length scale $\ell=\ell_{s}$, their intrinsic length

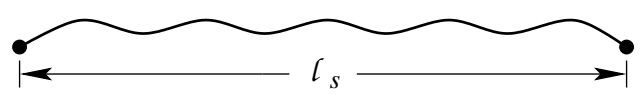

whose square is inversely proportional to the string tension and such that strings reduce to point particles in the limit $\ell_{s} \rightarrow 0$. Together with the string coupling constant, the string length $\ell_{s}$ dynamically determines the Planck scale of the target spacetime in which the strings live.

It was realized from early analyses of very high-energy string scattering amplitudes [31, 32] that, in order to prevent gravitational collapse, the objects in spacetime seen by strings must obey a modification of the Heisenberg uncertainty relation given by

$$
\Delta x \geq \frac{\hbar}{2} \frac{1}{\Delta p}+\frac{\ell_{s}^{2}}{2 \hbar} \Delta p
$$

At low energies this agrees with the standard phase space quantization, but for energies $E \gg \hbar c / \ell_{s}$ it implies that the extent of an observed object grows linearly with its momentum. Varying the right-hand side of the uncertainty relation (2.14) shows that it is minimized at $\Delta p=\hbar / \ell_{s}$, and substituting this back into (2.14) shows that the minimum spatial resolution seen by a string probe is

$$
(\Delta x)_{\min }=\ell_{s} .
$$

This simply means that strings cannot probe distances below their intrinsic size, and this fact was used to suggest very early on that the concept of spacetime changes its meaning below the Planck scale, due to the non-locality of string interactions.

Via some basic kinematical conformal invariance arguments, it was subsequently realized that in fact the target space probed by strings must also be subjected to spacetime uncertainty relations [33]

$$
\Delta x \Delta t \geq \frac{\ell_{\mathrm{P}}^{2}}{c},
$$

analogous to what was observed in $\S 2.4$. Relations such as these can be derived dynamically by looking at non-perturbative degrees of freedom in string theory. This was done by noting that 
sub-Planckian scales in string theory are probed by D-branes [34], whose dynamics thus enable one to provide a microscopic derivation of these and other modified uncertainty relations [35, 36]. A D-brane is a boundary condition for open strings. We can regard D-branes as hypersurfaces in spacetime

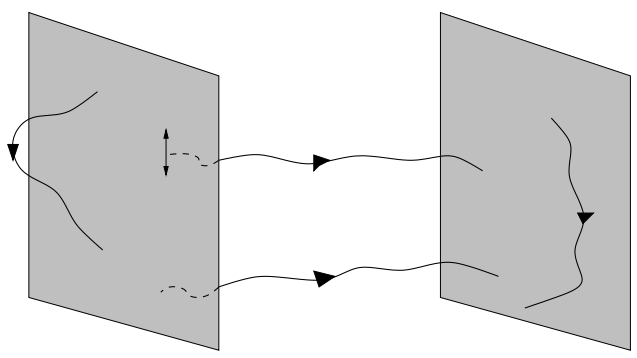

onto which the endpoints of open strings attach. The quantum dynamics of open strings induce fields which live on the branes. These include, among many others, Chan-Paton gauge fields $A_{\mu}$, scalar fields $X^{m}$ describing the location of the D-brane in spacetime, and also fermion fields $\psi$ in the supersymmetric case. In the low-energy limit, the spacetime dynamics of the open strings are described by an effective field theory living on the D-branes, which dynamically describes how the shape and size of the D-brane fluctuates in spacetime. In particular, by considering string theory with D-branes and certain background "magnetic" fields $B$ induced by the closed string sector, the low-energy limit is described by a field theory on a noncommutative space [37, 38], via a mechanism analogous (though somewhat more involved) to that described in $§ 2.5$. It is this D-brane field theory that we will be interested in for the remainder of this survey.

The derivation just sketched is what led to the huge intensity of research starting at the end of the last millenium, and has today developed into its own branch of physics called "noncommutative field theory". It gives an explicit realization of the manner in which worldvolume field theories are altered by quantum gravitational effects. Since the closed string sector induces the geometric fields of the target spacetime, D-branes can interact gravitationally with one another by exchanging closed strings. This couples the D-brane field theory to gravity, and will be one of the ways in which field theories on quantized spaces are intimately connected to gravity.

\section{$3 \quad$ Field theory on quantized spacetimes}

In this section we will describe some aspects of the perturbative dynamics of the noncommutative quantum field theories we derived in the previous section. Later on we will see how these novel features can be reinterpreted in the context of quantizing gravity directly from noncommutative field theory, and also in terms of deformed dispersion relations such as (2.3) and (2.5). In this section we will only consider scalar fields, defering the discussion of gauge fields to the next section. Henceforth, unless otherwise indicated, we shall work in euclidean signature and with natural units in which $\hbar=c=1$. We will always assume that the antisymmetric matrix $\theta^{\mu \nu}$ is invertible.

\subsection{Formalism}

Let us begin by describing the basic aspects of formulating noncommutative quantum field theory, as a deformation of ordinary field theory (see $[1,2]$ for further details). The noncommutative deformation is implemented by replacing the usual pointwise product of a pair of fields $\phi$ and $\psi$ by the "star-product"

$$
\phi(x) \psi(x) \longrightarrow(\phi \star \psi)(x)=\phi(x) \psi(x)+O(\theta, \partial \phi \partial \psi) .
$$


The star-product defines an associative but noncommutative multiplication on the space of fields, which deforms the usual product that is recovered at $\theta=0$. We will regard it as containing infinitely-many bi-derivative terms. There are several ways to express the star-product, depending on the domain of fields used. For most of our considerations below, we will use an integral representation of the star-product, which makes it a non-local operation. The two representations coincide on analytic fields. Here we shall only need its simplest incarnation. Denoting by $\tilde{\phi}$ the Fourier transform of the field $\phi$, the momentum space version of (3.1) modifies the Fourier convolution product as

$$
\tilde{\phi}(k) \tilde{\psi}(q) \longrightarrow \tilde{\phi}(k) \tilde{\psi}(q) \mathrm{e}^{\mathrm{i} k \times q} \quad \text { with } \quad k \times q=\frac{1}{2} k_{\mu} \theta^{\mu \nu} q_{\nu} .
$$

An easy calculation shows that this definition gives the required commutation relations

$$
\left[x^{\mu}, x^{\nu}\right]_{\star}=x^{\mu} \star x^{\nu}-x^{\nu} \star x^{\mu}=\mathrm{i} \theta^{\mu \nu} .
$$

Given any ordinary field theory with action $S$, one obtains a noncommutative field theory simply by replacing all products of fields occuring in $S$ with star-products. This deformation only affects the interactions of fields, owing to the identity

$$
\int \phi \star \psi=\int \phi \psi
$$

which is straightforwardly derived via integration by parts. This property is particular to the relatively simple noncommutative spaces we have restricted to, and it is another reflection of the flatness of momentum space (equivalently, the invariant line element $\mathrm{d} s^{2}$ is undeformed). It implies that the underlying free field theory is unaltered by the effects of noncommutativity. This is one reason why the quantum dynamics of these noncommutative field theories are tractable, at least in perturbation theory.

\subsection{UV/IR mixing}

Let us now describe the most notorious problem which arose in some of the first studies of perturbative noncommutative field theory. Consider, for example, the simplest instance of a real scalar $\lambda \phi^{\star n}$ theory. The interaction vertices are given by the Feynman rules

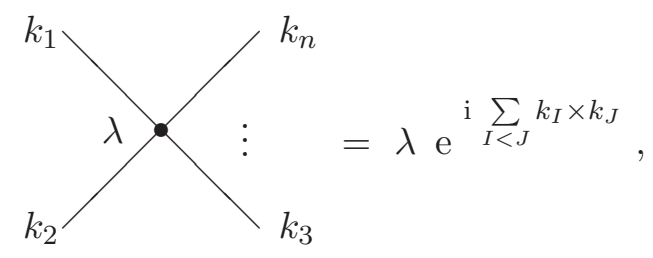

with the usual momentum conservation $k_{1}+k_{2}+\ldots+k_{n}=0$ since the noncommutative space (and the noncommutative field theory) is translationally invariant. The phase factors in these interactions become effective at energies $E$ with $E \sqrt{\theta} \gg 1$.

In contrast to the commutative case, the vertex (3.5) depends on the ordering of the momenta $k_{I}$. However, it depends only on their cyclic ordering. In analogy with how one handles the perturbative dynamics of multi-colour field theories, it is convenient to represent each Feynman diagram by fattening its lines and making the diagram into a ribbon graph. Then the set of all graphs comes with a notion of planarity. Planar graphs are those which can be drawn on the plane without crossing lines. They coincide with the ordinary graphs at $\theta=0$, times possible phase factors depending only on the momenta of the external legs [39, 40]. This shows that noncommutativity does not tame the ultraviolet divergences of quantum field theory as originally hoped, at least for 
this simple class of noncommutative spaces, and the noncommutative field theory contains at least the same divergences.

What is even worse is the behaviour of the non-planar graphs, those which cannot be drawn on the plane without crossing lines. In these diagrams, the phase factors of (3.5) depend on the virtual momenta of internal loops. Although these phase factors succeed in damping out the highenergy behaviour of the graphs through rapid oscillations and would seem to provide a natural ultraviolet cutoff $1 / \theta \cdot k$, they become ineffective for vanishing momenta and the original ultraviolet divergences reappear as infrared divergences. This phenomenon is called UV/IR mixing [41]. It leads to severe problems when one-loop non-planar diagrams occur as subgraphs in two-loop and higher order graphs, giving uncontrollable divergences which prevent the renormalization of higher orders in perturbation theory [42]. In other words, the introduction of an ultraviolet cutoff $\Lambda$ in the field theory induces an effective infrared cutoff

$$
\Lambda_{0}=\frac{1}{\theta \Lambda} .
$$

This mixing prohibits the use of standard renormalization schemes, such as the wilsonian approach, which require a clear separation of energy scales. It follows that the field theory cannot be renormalized. This problem plagued noncommutative field theory for many years.

In order to understand how to overcome this serious problem, it is useful to briefly look at the physics underlying the UV/IR mixing phenomenon. The mixing is due to the inherent non-locality of the quantum field theory. If $\phi$ and $\psi$ are fields which are both supported in a region of small size $\Delta \ll \sqrt{\theta}$, then their star-product $\phi \star \psi$ is non-zero in a large region of size $\theta / \Delta$. A drastic example of this occurs when both fields are localized at a point, as then their interaction is supported on all of space due to the identity

$$
\delta(x) \star \delta(x)=\frac{1}{\operatorname{det}(\pi \theta)} .
$$

This means that the quanta governing the interactions of noncommutative field theory in the ultraviolet must also be non-local extended objects which are very different from the pointlike quanta encountered in ordinary quantum field theory.

The nature of these new degrees of freedom can be understood via an elementary calculation using (3.3) and the Baker-Campbell-Hausdorff formula which gives the identity

$$
\mathrm{e}^{\mathrm{i} k \cdot x} \star \phi(x) \star \mathrm{e}^{-\mathrm{i} k \cdot x}=\phi\left(x^{\mu}-\theta^{\mu \nu} k_{\nu}\right) .
$$

We interpret this to mean that the ultraviolet dynamics, in the regime $E \gg \theta^{-1 / 2}$, of the fields behave as if they were supported on extended, rigid rods whose size is proportional to their momentum. These are "dipoles" with dipole moment

$$
\Delta x^{\mu}=\theta^{\mu \nu} k_{\nu}
$$

and they interact by joining at their ends $[43,44]$. This is analogous to the electron-hole bound states in a strong magnetic field, as in the systems described in $\S 2.5$, and it also agrees with the modified uncertainty relation (2.14) derived from string theory. Thus in the ultraviolet the natural degrees of freedom are dipoles. On the other hand, the infrared dynamics, in the regime $E \ll \theta^{-1 / 2}$ where the effects of noncommutativity are negligible, is mediated by the elementary quantum fields $\phi$ themselves, with pointlike momenta $k_{\mu}$. This structure is in fact suggested directly by the commutation relations (2.7), which lead to a noncommutative spacetime at high energies and an almost commutative spacetime in the infrared regime. Although completely different in their respective characteristics, it has been suggested that there is a UV/IR "duality" relating the 
dynamics in the two regimes [45]. Evidence for this duality is provided via perturbative calculations using explicit operators

$$
W_{k}[\phi]=\int \exp (\mathrm{i}|k| \phi(x))
$$

which create the noncommutative dipole degrees of freedom.

This simple physical picture suggests that the origin of the UV/IR mixing problem is associated with the asymmetry between extended and pointlike degrees of freedom in the different regimes of the quantum field theory. One thereby seeks a covariant version of the field theory which renders the ultraviolet and infrared regimes indistinguishable [46], and hence makes the UV/IR "duality" above into a true symmetry of the model. This covariantization turns the infrared degrees of freedom into extended objects by replacing their pointlike momenta with the "Landau" momenta

$$
k_{\mu} \longmapsto K_{\mu}=k_{\mu}+B_{\mu \nu} x^{\nu},
$$

analogously to the way in which the ultraviolet degrees of freedom extend into dipoles. The additional non-degenerate constant antisymmetric matrix $B_{\mu \nu}$ is in general independent of $\theta^{\mu \nu}$ and can be interpreted as a "magnetic" background. Assuming that $\left(x^{\mu}, k_{\nu}\right)$ form a canonical pair, the new momenta $K_{\mu}$ generate a "noncommutative momentum space" with the commutation relations

$$
\left[K_{\mu}, K_{\nu}\right]=2 \text { i } B_{\mu \nu}
$$

in exactly the same in which noncommutative space arose in the Landau problem of $\S 2.5$ [8]. The covariant version of a noncommutative complex scalar field theory thus consists in replacing the hamiltonian of the usual kinetic term with the generalized Landau hamiltonian defined by the square of gauge covariant derivatives in the magnetic background according to (3.11).

The real version of the duality covariant model is known as the Grosse-Wulkenhaar model [47]. Drawing on the mapping between the Landau hamiltonian and the harmonic oscillator hamiltonian, one considers a real $\lambda \phi_{2}^{\star 4}$-theory in a background harmonic oscillator potential, defined by modifying the scalar field kinetic term via the replacement

$$
\partial_{\mu}^{2} \longmapsto \partial_{\mu}^{2}+\frac{\omega^{2}}{2} \tilde{x}_{\mu}^{2} \quad \text { with } \quad \tilde{x}_{\mu}=2 \theta_{\mu \nu}^{-1} x^{\nu}
$$

One then shows [46] that the full quantum field theory is symmetric under symplectic Fourier transformation of the fields which interchanges

$$
k_{\mu} \leftrightarrow \tilde{x}_{\mu}
$$

Modifying the noncommutative field theory in this way gives the new free propagators the correct decay behaviour, due to the confining nature of the harmonic oscillator potential, that enables one to apply wilsonian renormalization in a suitable duality-invariant basis of fields. Note that while any confining potential could in principle be used instead in (3.13) to give an effective infrared cutoff, the harmonic oscillator potential is singled out as the unique one which induces the UV/IR duality.

\subsection{Renormalization}

Let us now discuss some consequences of the UV/IR duality. The main success of the duality covariant model is that it is renormalizable to all orders in the $\phi^{\star 4}$ coupling constant $\lambda$ [47]. The proof is obtained by an exact mapping of the field theory onto a (infinite-dimensional) matrix 
model, which naturally arises due to the infinite degeneracy of Landau levels that provides a twoindex basis set of complete Landau wavefunctions for the expansion of the noncommutative fields. The duality-invariant cutoff is then naturally taken to be the matrix size $N$, and the wilsonian approach can be applied to the truncated model as $N$ varies. This matrix model possesses several interesting characteristics [48]. For example, the $N \times N$ matrix model is related to an integrable KP-hierarchy and, for certain choices of parameters, it is exactly solvable in the limit $N \rightarrow \infty$ as the cutoff is removed. This remarkable reformulation of noncommutative field theory without any reference to a background spacetime has no analog in ordinary quantum field theory. It is the crux of many important investigations and will arise again in the next section.

The point $\omega=1$ in parameter space is special and is called the self-dual point, because there the quantum field theory is completely invariant under the UV/IR duality (without any rescalings). This point corresponds to $\theta=2 B^{-1}$ where the noncommutativity is induced by the magnetic background of $\S 3.2$, as it happens in the Landau problem (see (2.13)). At this point, a remarkable property is found $[49,50]$. The beta-functions for both the coupling constant $\lambda$ and the oscillator parameter $\omega$ vanish to all orders of perturbation theory. This implies that the renormalized coupling flows to a finite bare coupling. The novel mechanism at work is that the wavefunction renormalization compensates exactly the coupling constant renormalization, such that the interaction $\lambda \phi^{\star 4}$ is invariant. In addition, the renormalized oscillator parameter flows to the value $\omega=1$.

This boundedness of the renormalization group flow has the following remarkable significance. To understand its implications, we need only recall that a particle in the flow of a quadratic vector field goes to infinity in a finite time. A similar feature was discovered in the early days of quantum electrodynamics, where it was observed that the coupling constant flows to infinity at very high, but finite, energies. Thus the high-energy perturbation series does not make sense, due to spurious poles that appear in a partial resummation of the expansion which is possible in this energy regime. The non-perturbative degrees of freedom corresponding to these poles is called the Landau ghost or renormalons. The Landau ghost also plagues ordinary $\phi^{4}$-theory in four dimensions, and it hinders a non-perturbative definition of the quantum field theory. Were it not for the discovery of asymptotic freedom in quantum chromodynamics, the Landau ghost might have disseminated quantum field theory. What is remarkable is that also in the duality covariant noncommutative field theory, there are no renormalons. But here the field theory is not asymptotically free, rather it is asymptotically safe.

For these reasons, it is strongly believed that a non-perturbative completion of these noncommutative quantum field theories is possible, and intense research on this matter has been pursued over the past few years. See [5] and references therein for a discussion of these matters. From these perspectives, the duality covariant models may teach important lessons for the consistent treatment of ordinary quantum field theories. This programme would require a proper understanding of the meaning of the parameters $\theta$ and $\omega$ at observable energies, and also a thorough understanding of how to pass to our physical $3+1$-dimensional spacetime. The analytic continuation of the duality covariant field theories to Minkowski signature has been recently carried out in [51], but the renormalization of noncommutative quantum field theory in Minkowski spacetime is still very much in its infancy. In particular, a simple physical explanation of electric-type noncommutativity like the lowest Landau level projection of $\S 2.5$ is currently lacking.

$\mathrm{UV} / \mathrm{IR}$ mixing is also known to occur on more complicated noncommutative spaces, such as the $\kappa$-deformed space discussed in $\S 2.2$ [52]. The precise meaning of the duality covariance in these and other instances is not very well understood, and it may be related to some of the wellknown position-momentum type quantum group dualities. The UV/IR duality has been interpreted recently in [53] in terms of metaplectic representations of the Heisenberg group, where the GrosseWulkenhaar model on solvable symmetric spaces is formulated. 


\section{Noncommutative gauge theory of gravity}

Gauge theories on quantized spacetime are of particular interest for a variety of reasons. They enable one to attempt to formulate more realistic physical models which may provide a means for comparing with measurable signatures of spacetime noncommutativity. This aspect will be discussed in the next section. They are also the natural field theories that arise on D-branes subjected to external backgrounds [38], in the context discussed in $§ 2.6$. Given their role in string theory, it is natural to expect that they may canonically couple to gravitational degrees of freedom, and hence their quantization may teach us something about quantum gravity. This is the theme which we shall have in mind in this section. We will see that the UV/IR mixing phenomenon of the previous section has a beautiful gravitational avatar in this context.

\subsection{Gauge interactions}

The action for a $U(N)$ gauge field $A_{\mu}(x)$ in noncommutative Yang-Mills theory is given by

$$
S=-\frac{1}{4 g^{2}} \int \operatorname{Tr} F_{\mu \nu}^{2}
$$

with the field strength tensor

$$
\begin{aligned}
F_{\mu \nu} & =\partial_{\mu} A_{\nu}-\partial_{\nu} A_{\mu}-\mathrm{i}\left[A_{\mu}, A_{\nu}\right]_{\star} \\
& =\partial_{\mu} A_{\nu}-\partial_{\nu} A_{\mu}-\mathrm{i}\left[A_{\mu}, A_{\nu}\right]+O\left(\theta,(\partial A)^{2}\right) .
\end{aligned}
$$

This model thus gives a modification of ordinary gauge theory by infinitely many higher-derivative interaction terms. The gauge invariance in this model is manifested by the invariance of (4.1) under the "star-gauge transformations"

$$
A_{\mu} \longmapsto U \star A_{\mu} \star U^{-1}+\mathrm{i} U \star \partial_{\mu} U^{-1} \quad \text { with } U \star U^{\dagger}=U^{\dagger} \star U=1 .
$$

It follows that the gauge symmetry of noncommutative Yang-Mills theory contains an intricate mixing of both colour and spacetime transformations. It generates an infinite-dimensional unitary symmetry group isomorphic to $U(\infty)$, as will become more apparent below when we consider a certain matrix model formulation of this gauge theory. Geometrically, star-gauge transformations generate certain "deformed" canonical transformations with respect to the Poisson structure induced by the noncommutativity matrix $\theta^{\mu \nu}[54]$.

Let us briefly summarize some of the novel features of noncommutative gauge theories that distinguish them from their commutative counterparts. Since the group $S U(N)$ does not close under the star-product, because $\operatorname{det}(\phi \star \psi) \neq \operatorname{det}(\phi) \star \operatorname{det}(\psi)$ in general, the $U(1)$ sector cannot be decoupled from the $S U(N)$ sector [55]. The $U(1)$ coupling constant flows, and its beta-function is found to agree precisely with the beta-function of planar (large $N) S U(N)$ Yang-Mills theory [56] (the reason for this coincidence will become evident below). Wilson loops have been observed to display a phase structure $[57,58]$. They obey the usual area law for small area loops, where the effects of noncommutativity become negligible, while large area loops acquire an imaginary Aharanov-Bohm phase with respect to the magnetic field $B=1 / \theta$ (again in agreement with (2.13)). UV/IR mixing is present but occurs through a logarithmic dependence [59] (in contrast to

the power-law dependence in scalar field theories) and only in the $U(1)$ sector [55]. This produces a strange infrared behaviour of the "photon" in these gauge theories, which may be compared to the high-energy behaviour of gamma-ray bursts analogously to what we described in $\S 2.2$. We will return to this point in the next section. The duality covariant version of noncommutative gauge theory is not presently known. 
Let us also briefly mention a key tool for the analysis of noncommutative Yang-Mills theory, the celebrated Seiberg-Witten map [38]. This transformation provides a one-to-one correspondence between commutative and noncommutative gauge orbits of gauge fields $A_{\mu}$, and is valid at length scales much longer than the scale of noncommutativity $\sqrt{\theta}$. When applied to the action (4.1), it defines an ordinary gauge theory which is "dual" to noncommutative Yang-Mills theory. The dual gauge theory is not a Yang-Mills theory, but contains many more higher order gauge vertices. It has been utilized in a variety of contexts, and will play a crucial role in our discussion later on. It has proved to be particularly useful for producing "phenomenological" models which could test the existence of spacetime noncommutativity in nature, for example noncommutative extensions of the standard model which can probe physics beyond the standard model [60]. We will also return to this point in the next section.

\subsection{Gravity in noncommutative gauge theories}

It was realized early on that gravity is naturally contained in the dynamics of noncommutative gauge theory, though the precise mechanism was not initially clear. This is due to the spacetime transformation properties of the gauge symmetry in these models. The key observation is that spacetime translations of noncommutative gauge fields are equivalent to gauge transformations. Using the identity (3.8), the star-gauge transformation (4.3) by the star-unitary field

$$
U(x)=\mathrm{e}^{\mathrm{i} \theta_{\mu \nu}^{-1} a^{\mu} x^{\nu}}
$$

is found to be

$$
A_{\mu}(x) \longmapsto A_{\mu}(x+a)-\theta_{\mu \nu}^{-1} a^{\nu} .
$$

The constant shift in (4.5) drops out of the noncommutative field strength tensor (4.2), and hence the translational symmetry is a gauge symmetry [61]. This means that noncommutative gauge theories provide "toy models" of general relativity, and may possibly enable one to construct diffeomorphism invariant field theories.

The key to making this observation more precise is to be able to promote the global translation symmetry to a local gauge symmetry, and to extend the gauging to the full Poincaré symmetry of four-dimensional spacetime. There have been various suggestions on how to obtain UtiyamaKibble type gauge theories of gravity along these lines. For example, certain dimensional reductions of noncommutative Yang-Mills theory from ten dimensions to four dimensions naturally induce deformations of a Poincaré gauge theory of gravity, owing to the occurence of teleparallism in noncommutative gauge theory $[62,3]$. General relativity on noncommutative spacetime has also been constructed by gauging the twist-deformed Poincaré symmetry [63]. Deformations of gravity can moreover be induced from a noncommutative gauge theory with position-dependent noncommutativity $\theta^{\mu \nu}(x)$ using the Seiberg-Witten map [64]. We will shortly see how an analogous procedure can be used to extract general relativity more precisely from the dynamics of noncommutative gauge fields. Noncommutative gauge theories of gravity based on the Lorentz group $S L(2, \mathbb{C})$ with complex vierbein have been considered in $[65,66]$

\subsection{Spacetime disappears}

The crux of the identification of gravitational dynamics is the background independent formulation of noncommutative gauge theory [38], which allows a reformulation of the gauge dynamics without any reference to a background spacetime. For this, we introduce the "covariant coordinates" [67]

$$
X_{\mu}=\theta_{\mu \nu}^{-1} x^{\nu}+A_{\mu}
$$


in terms of which the noncommutative field strength tensor (4.2) can be written as

$$
F_{\mu \nu}=-\mathrm{i}\left[X_{\mu}, X_{\nu}\right]_{\star}+\theta_{\mu \nu}^{-1}
$$

This rewriting of the gauge fields again exploits the translation-generating property (3.8) to write derivatives as inner automorphisms of the algebra of fields. We may thus reformulate noncommutative Yang-Mills theory with action (4.1) entirely using the operators $X_{\mu}$ which can be regarded now as abstract objects of an infinite-dimensional matrix algebra, without any reference to a spacetime dependence.

The noncommutative gauge theory thereby becomes a matrix model with action

$$
S=-\frac{1}{4 g^{2}} \operatorname{Tr}\left(-\mathrm{i}\left[X_{\mu}, X_{\nu}\right]+\theta_{\mu \nu}^{-1}\right)^{2},
$$

where the infinite-dimensional trace implicitly hides the integration over spacetime. This action actually arises in an independent context as a twisted reduced model. It is the dimensional reduction of ordinary Yang-Mills theory to a point, i.e. with gauge fields which do not depend on the spacetime coordinates, with the constant shift $\theta_{\mu \nu}^{-1}$ the "twist". It is related to the IKKT matrix model for the non-perturbative dynamics of Type IIB superstrings [68]. The equations of motion derived by varying (4.8) are

$$
\left[X_{\mu},\left[X_{\mu}, X_{\nu}\right]\right]=0
$$

In particular, the vacuum solution is given by matrices $X_{\mu}$ satisfying

$$
\left[X_{\mu}, X_{\nu}\right]=-\mathrm{i} \theta_{\mu \nu}^{-1}
$$

which gives the absolute minimum value of the action (4.8). This solution is obtained from (4.6) with $A_{\mu}=0$, and so in this context noncommutative spacetime arises as a dynamical effect in the matrix model, analogously to the way it did in $\S 2.3$. The decomposition (4.6) then identifies the gauge field degrees of freedom as fluctuations around the original noncommutative spacetime coordinates $x^{\mu}$.

The vacuum state is defined by a Heisenberg algebra (4.10), which has only infinite-dimensional representions. To have a constructive definition of noncommutative gauge theory, we would like to define the natural regularization (as in $\S 3.3$ ) by cutting off the matrix rank at some finite value $N$. As formulated, this is not possible because the vacuum equation (4.10) has no finite rank $N \times N$ solutions about which we can expand to uncover the dynamics of our original gauge theory. A non-perturbative definition was proposed in [69] based on the simple observation that while the Heisenberg commutation relations (4.10) do not admit any finite-dimensional representations, its "exponentiated version" (the Weyl algebra) does for certain values of the noncommutativity parameter. Thus instead of the hermitean degrees of freedom $X_{\mu}$, one considers a unitary matrix model involving $N \times N$ unitary matrices $U_{\mu}$ with action

$$
S=-\frac{1}{4 g^{2}} \sum_{\mu \neq \nu} \mathrm{e}^{-2 \pi \mathrm{i} Q_{\mu \nu} / N} \operatorname{Tr}\left(U_{\mu} U_{\nu} U_{\mu}^{\dagger} U_{\nu}^{\dagger}\right) .
$$

The matrix model (4.8) is recovered by setting

$$
U_{\mu}=\mathrm{e}^{\mathrm{i} a X_{\mu}}
$$

and expanding to leading orders in the lattice spacing $a$. This identifies the noncommutativity parameter as

$$
\theta_{\mu \nu}^{-1}=\frac{2 \pi Q_{\mu \nu}}{N a^{2}}
$$


The non-trivial vacuum state is now given in terms of the well-known $S U(N)$ 't Hooft twist-eating solutions $(N \times N$ clock and shift matrices $)$.

The matrix model with action (4.11) is known as the twisted Eguchi-Kawai model. It can also be independently derived as the one-plaquette reduction of Wilson's lattice gauge theory with background 't Hooft flux $Q_{\mu \nu}$ (the "twist"). It was originally proposed as a close relative of the planar $N \rightarrow \infty$ limit of multi-colour Yang-Mills theory, whose dynamics are more tractable both analytically and numerically. In the present context, this same matrix model admits a concrete finite $N$ interpretation as a noncommutative version of lattice gauge theory, with the matrix rank $N$ giving the finite size of the lattice [69]. The automatic requirement of a (periodic) lattice of finite size for $N$ finite is a non-perturbative manifestation of UV/IR mixing. The unitary matrix model has proven to be useful for numerical studies of noncommutative gauge theory (see e.g. [70] and references therein).

There are two distinct scaling limits of the matrix model (4.11) with $N \rightarrow \infty, a \rightarrow 0$, which explains the intimate relationship between the noncommutative and planar Yang-Mills theories that we noticed earlier. Taking the large $N$ limit first sends $\theta \rightarrow \infty$, and gives the 't Hooft limit of large $N$ Yang-Mills theory (the limit $\theta \rightarrow \infty$ is also known to kill all non-planar graphs of the noncommutative field theory [2]). On the other hand, we can consider a special double-scaling limit in which the combination $\sqrt{N} a^{2}$ is kept finite. Then the noncommutativity $\theta$ remains finite and one recovers noncommutative Yang-Mills theory.

\subsection{Gravitational dynamics and UV/IR mixing}

The precise origin of gravity in noncommutative gauge theory is through a process of emergent gravity, as first observed in [71, 72] and clarified in a systematic manner in [73]. One considers non-vacuum solutions

$$
\left[X^{\mu}, X^{\nu}\right]=\mathrm{i} \theta^{\mu \nu}(x)
$$

of the equations of motion (4.9), with position dependent noncommutativity parameters. Via the Seiberg-Witten map, this describes a dynamical quantum spacetime. In this setting, gravity is related to the quantum fluctuations $X^{\mu}$ of spacetime at the Planck scale. Conversely, noncommutative field theory arises as field dependent fluctuations of spacetime geometry determined by the Poisson bivector $\theta^{\mu \nu}(x)$ in (4.14). This observation can be used to clarify many of the novel features of noncommutative gauge theory that we described in $\S 4.1$. For example, the $U(1)$ "photon" is really a graviton, which defines a non-trivial geometric background coupled to $S U(N)$ gauge fields. This provides a natural physical explanation for the $U(1) \leftrightarrow S U(N)$ entanglement. The resulting gravitational theory is similar to general relativity for weak curvature. It suggests a new approach to the quantization and unification of gravity with gauge theory. In particular, the flat space solution (4.10) is stable at one-loop order.

The formalism also provides a beautiful physical explanation of the UV/IR mixing problem in noncommutative field theory. For this, consider the infrared dynamics for momenta $k$ in the regime

$$
k<\Lambda<\Lambda_{\mathrm{NC}}=\frac{1}{\sqrt{\theta}} .
$$

In [74], the one-loop effective action for noncommutative gauge fields coupled to dynamical adjoint scalar fields is computed, involving a careful treatment of the UV/IR mixing terms. By using wellknown heat kernel expansions for scalar field kinetic laplacian operator, one arrives at an induced Einstein-Hilbert action of the form

$$
S=\int \sqrt{g}\left(c_{1} \Lambda^{4}+c_{2} \Lambda^{2} R(g)+O(\log \Lambda)\right) .
$$


Thus UV/IR mixing gives a non-renormalizable gravitational sector, with the ultraviolet cutoff $\Lambda$ of the gauge theory related to the newtonian gravitational constant $G$ according to (4.16). This provides a concrete physical explanation for the non-renormalizability of noncommutative field theories caused by UV/IR mixing.

The dipole quanta in the ultraviolet regime are in this case created by the open Wilson line

operators $\operatorname{Tr}\left(\mathrm{e}^{\mathrm{i} k \cdot X}\right)[75,76,45]$, and the physical interpretation given in $\S 3.2$ agrees with the way in which these operators are argued to couple to gravitational degrees of freedom in string theory [77]-[80]. The process described here is similar to the mechanism of UV/IR mixing that occurs on a D-brane in a background $B$-field, which is related to tachyonic instabilities arising from the exchange of closed string modes in the bulk [81, 82]. It is complementary to the derivation of the newtonian force law [83] and supergravity graviton propagators [84] from four-dimensional maximally supersymmetric noncommutative Yang-Mills theory.

\section{Signatures of spacetime noncommutativity}

In this final section we will briefly indicate how spacetime noncommutativity may be observed or measured explicitly in experiment. There are many proposals for how this may be achieved, none of which are entirely conclusive. Of course, much of the issue concerns the magnitude of the effects of noncommutativity, and the hope is that there exist physical processes for which the effective scale of noncommutativity lies within the present day experimental energy range (see $\S 1$ ). Here we shall not attempt an exhaustive survey, but rather just highlight a selection of ideas which have been put forward, in order to give a flavour for some of the main issues involved. In particular, we will connect UV/IR mixing with some of the results of $\S 2$.

\subsection{Violations of Lorentz invariance and causality}

Noncommutative field theories provide examples of both Lorentz invariance and causality violating models, thus destroying the two pillars of relativistic quantum field theory. In four spacetime dimensions, the rank two antisymmetric tensor $\theta^{\mu \nu}$ provides a directionality

$$
\boldsymbol{\theta}_{i}=\epsilon_{i j k} \theta^{j k}
$$

in space. It follows that noncommutative field theory is not invariant under rotations or boosts of localized field configurations within a fixed observer inertial frame. In string theory, this symmetry breaking is due to the expectation value of a background supergravity field. A notion of Lorentz invariance may be recovered through a twist-deformation of the action of the Lorentz group generators on the noncommutative fields $[85,86]$.

There is also no sharply localized light-cone (due to spacetime uncertainty relations), so causality is violated and signals can travel faster than the speed of light in quantized spacetime. A notion of localization of noncommutative quantum fields on "light-wedges" has been recently developed in [87]. Thus the spin-statistics theorem and the CPT theorem do not (necessarily) hold in noncommutative field theory. We can now try to find physically measurable processes which would otherwise be forbidden by the usual rules of relativistic quantum field theory. If observed in experiment, they would vindicate the need for noncommutative field theory at high (but measurable) energies.

Let us now give a taste of some of the processes that one may attempt to analyse. In nonabelian gauge theories, such as noncommutative deformations of quantum chromodynamics or the 
standard model, the S-matrix for some processes violates Lorentz invariance. For example, the Feynman diagram

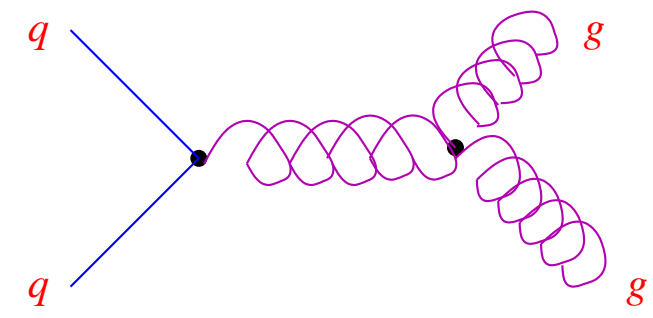

is not Lorentz invariant, because the propagator has a frame dependence on the directionality $\boldsymbol{\theta}^{0}{ }_{i}=\theta^{0 i}[88]$. This is reminescent of Yang's theorem in the commutative case, which forbids certain diagrams due to Lorentz symmetry violation (despite the Lorentz invariance of the local tree-level lagrangian). However, such processes are allowed in Lorentz-violating theories such as noncommutative gauge theory. If even a small amplitude for such a scattering process could be observed, it would signal evidence for noncommutativity and put bounds on the magnitude of the parameter $\theta$. For example, a comparison of the leading terms in a noncommutative deformation of the standard model with known Lorentz-violating (but CPT symmetric) extensions of the standard model estimate [89]

$$
\theta \leq\left(10^{-25} \mathrm{~cm}\right)^{2} .
$$

Other Lorentz-violating processes, which have been studied in the context of noncommutative extensions of the standard model, include $Z^{0} \rightarrow \gamma \gamma$ [90, 91] and decays of quarkonia [92].

Violations of causality can be most readily observed by looking at signatures for spin-statistics violation extracted from atomic transitions in quantum electrodynamics. One can compare with experimental results that put limits on the violation of the Pauli exclusion principle in nucleon systems, based on non-observational transitions from states which are allowed to occupied levels which are forbidden by the Pauli principle. For example, comparison with neutrino data from Gran Sasso or Super-Kamiokande gives a strong bound [88]

$$
\theta \leq\left(10^{-24} \mathrm{~cm}\right)^{2} .
$$

Some simpler analyses of atomic transitions in noncommutative quantum mechanics can also be carried out, for instance of the Lamb shift in hydrogen [93], and the leading corrections due to noncommutativity compared with experiment.

\subsection{Dispersion relations and $U V / I R$ mixing}

The presence of UV/IR mixing terms in the one-loop effective actions of noncommutative field theories leads to modified (photon or scalar) dispersion relations analogous to those discussed in $\S 2.2$ and $\S 2.3$. They take the general form

$$
E^{2}=\boldsymbol{p}^{2}+m^{2}+\Delta M^{2}\left(\frac{1}{p \theta}\right)
$$

One can compare these dispersion relations with experiments in the energy range

$$
\Lambda_{0}<E<\Lambda=\frac{1}{\theta \Lambda_{0}}
$$

where $\Lambda_{0}$ is the phenomenological infrared scale. For example, a comparison with observational data from blazars estimates the noncommutativity parameter as [94, 95]

$$
\theta \geq\left(10^{13} \ell_{\mathrm{P}}\right)^{2}=\left(10^{-20} \mathrm{~cm}\right)^{2} .
$$

Analogous estimates from cosmic microwave background radiation data can be found in [96]. 


\section{Acknowledgments}

The author would like to thank the organisors of the XXIX Encontro Nacional de Física de Partículas e Campos, and in particular V. Rivelles, for a stimulating meeting and an enjoyable time in Brasil. This work was supported in part by the EU-RTN Network Grant MRTN-CT-2004005104 .

\section{References}

[1] M.R. Douglas and N.A. Nekrasov, "Noncommutative field theory," Rev. Mod. Phys. 73 (2001) 977-1029 [arXiv:hep-th/0106048].

[2] R.J. Szabo, "Quantum field theory on noncommutative spaces," Phys. Rept. 378 (2003) 207299 [arXiv:hep-th/0109162].

[3] R.J. Szabo, "Symmetry, gravity and noncommutativity," Class. Quant. Grav. 23 (2006) R199R242 [arXiv:hep-th/0606233].

[4] F. Müller-Hoissen, "Noncommutative geometries and gravity," AIP Conf. Proc. 977 (2008) 12-29 [arXiv:0710.4418 [gr-qc]].

[5] V. Rivasseau, "Noncommutative renormalization," Sém. Poincaré X (2007) 15-95 [arXiv:hepth/0702068].

[6] W. Bietenholz, "Cosmic rays and the search for a Lorentz invariance violation," arXiv:0806.3713 [hep-ph].

[7] R.J. Szabo, "Symmetries and renormalization of noncommutative field theory," AIP Conf. Proc. 917 (2007) 146-153 [arXiv:hep-th/0701224]

[8] R.J. Szabo, "Magnetic backgrounds and noncommutative field theory," Int. J. Mod. Phys. A 19 (2004) 1837-1862 [arXiv:physics/0401142].

[9] H.S. Snyder, "Quantized spacetime," Phys. Rev. 71 (1947) 38-41; "The electromagnetic field in quantized spacetime," Phys. Rev. 72 (1947) 68-71.

[10] C.N. Yang, "On quantized spacetime," Phys. Rev. 72 (1947) 874.

[11] J. Lukierski, H. Ruegg, A. Nowicki and V.N. Tolstoi, "q-deformation of Poincaré algebra," Phys. Lett. B 264 (1991) 331-338.

[12] S. Majid and H. Ruegg, "Bicrossproduct structure of $\kappa$-Poincaré group and noncommutative geometry," Phys. Lett. B 334 (1994) 348-354 [arXiv:hep-th/9405107].

[13] J. Kowalski-Glikman and S. Nowak, "Noncommutative spacetime of doubly special relativity theories," Int. J. Mod. Phys. D 12 (2003) 299-316 [arXiv:hep-th/0204245].

[14] G. Amelino-Camelia, "Relativity in spacetimes with short distance structure governed by an observer independent (Planckian) length scale," Int. J. Mod. Phys. D 11 (2002) 35-60 [arXiv:gr-qc/0012051]; "Testable scenario for relativity with minimum length," Phys. Lett. B 510 (2001) 255-263 [arXiv:hep-th/0012238].

[15] S. Ghosh, "A lagrangian for DSR particle and the role of noncommutativity," Phys. Rev. D 74 (2006) 084019 [arXiv:hep-th/0608206]. 
[16] S. Ghosh and P. Pal, "Deformed special relativity and deformed symmetries in a canonical framework," Phys. Rev. D 75 (2007) 105021 [arXiv:hep-th/0702159].

[17] J. Magueijo and L. Smolin, "Lorentz invariance with an invariant energy scale," Phys. Rev. Lett. 88 (2002) 190403 [arXiv:hep-th/0112090]; "Generalized Lorentz invariance with an invariant energy scale," Phys. Rev. D 67 (2003) 044017 [arXiv:gr-qc/0207085].

[18] L. Freidel and E.R. Livine, "Effective $3 D$ quantum gravity and noncommutative quantum field theory," Phys. Rev. Lett. 96 (2006) 221301 [arXiv:hep-th/0512113].

[19] N. Sasakura, "Spacetime uncertainty relation and Lorentz invariance," J. High Energy Phys. 0005 (2000) 015 [arXiv:hep-th/0001161].

[20] Y. Sasai and N. Sasakura, "The Cutkosky rule of three-dimensional noncommutative field theory in Lie algebraic noncommutative spacetime," J. High Energy Phys. 0906 (2009) 013 [arXiv:0902.3050 [hep-th]]; "Massive particles coupled with 2+1-dimensional gravity and noncommutative field theory," arXiv:0902.3502 [hep-th].

[21] R. Oeckl, "Braided quantum field theory," Commun. Math. Phys. 217 (2001) 451-473 [arXiv:hep-th/9906225].

[22] Y. Sasai and N. Sasakura, "Braided quantum field theories and their symmetries," Prog. Theor. Phys. 118 (2007) 785-814 [arXiv:0704.0822 [hep-th]].

[23] S. Doplicher, K. Fredenhagen and J.E. Roberts, "Spacetime quantization induced by classical gravity," Phys. Lett. B 331 (1994) 39-44; "The quantum structure of spacetime at the Planck scale and quantum fields," Commun. Math. Phys. 172 (1995) 187-220 [arXiv:hep-th/0303037].

[24] R.E. Peierls, "On the theory of diamagnetism of conduction electrons," Z. Phys. 80 (1933) 763-791.

[25] G.V. Dunne, R. Jackiw and C.A. Trugenberger, "Topological (Chern-Simons) quantum mechanics," Phys. Rev. D 41 (1990) 661-666.

[26] L. Susskind, "The quantum Hall fluid and noncommutative Chern-Simons theory," arXiv:hepth/0101029.

[27] A.P. Polychronakos, "Quantum Hall states as matrix Chern-Simons theory," J. High Energy Phys. 0104 (2001) 011 [arXiv:hep-th/0103013].

[28] S. Hellerman and M. van Raamsdonk, "Quantum Hall physics equals noncommutative field theory," J. High Energy Phys. 0110 (2001) 039 [arXiv:hep-th/0103179].

[29] B. Duplantier, "Conformal random geometry," in: Mathematical Statistical Physics, eds. A. Bovier, F. Dunlop, F. den Hollander, A. van Enter and J. Dalibard (Elsevier B.V., 2006), pp. 101-217 [arXiv:math-ph/0608053].

[30] E.V. Gorbar, S. Homayouni and V.A. Miransky, "Chiral dynamics in QED and QCD in a magnetic background and nonlocal noncommutative field theories," Phys. Rev. D 72 (2005) 065014 [arXiv:hep-th/0503028].

[31] D.J. Gross and P.F. Mende, "String theory beyond the Planck scale," Nucl. Phys. B 303 (1988) 407-454.

[32] D. Amati, M. Ciafaloni and G. Veneziano, "Can spacetime be probed below the string size?," Phys. Lett. B 216 (1989) 41-47. 
[33] T. Yoneya, "On the interpretation of minimal length in string theories," Mod. Phys. Lett. A 4 (1989) 1587-1595; "String theory and spacetime uncertainty principle," Progr. Theor. Phys. 103 (2000) 1081-1125 [arXiv:hep-th/0004074].

[34] M.R. Douglas, D.N. Kabat, P. Pouliot and S.H. Shenker, "D-branes and short distances in string theory," Nucl. Phys. B 485 (1997) 85-127 [arXiv:hep-th/9608024].

[35] M. Li and T. Yoneya, "D-particle dynamics and the spacetime uncertainty relation," Phys. Rev. Lett. 78 (1997) 1219-1222 [arXiv:hep-th/9611072].

[36] N.E. Mavromatos and R.J. Szabo, "Spacetime quantization from non-abelian D-particle dynamics," Phys. Rev. D 59 (1999) 064016 [arXiv:gr-qc/9807070]; "Matrix D-brane dynamics, logarithmic operators and quantization of noncommutative spacetime," Phys. Rev. D 59 (1999) 104018 [arXiv:hep-th/9808124].

[37] V. Schomerus, "D-branes and deformation quantization," J. High Energy Phys. 9906 (1999) 030 [arXiv:hep-th/9903205].

[38] N. Seiberg and E. Witten, "String theory and noncommutative geometry," J. High Energy Phys. 9909 (1999) 032 [arXiv:hep-th/9908142].

[39] T. Filk, "Divergences in a field theory on quantum space," Phys. Lett. B 376 (1996) 53-58.

[40] N. Ishibashi, S. Iso, H. Kawai and Y. Kitazawa, "Wilson loops in noncommutative Yang-Mills," Nucl. Phys. B 573 (2000) 573-593 [arXiv:hep-th/9910004].

[41] S. Minwalla, M. van Raamsdonk and N. Seiberg, "Noncommutative perturbative dynamics," J. High Energy Phys. 0002 (2000) 020 [arXiv:hep-th/9912072].

[42] M. van Raamsdonk and N. Seiberg, "Comments on noncommutative perturbative dynamics," J. High Energy Phys. 0003 (2000) 035 [arXiv:hep-th/0002186].

[43] M.M. Sheikh-Jabbari, "Open strings in a $B$-field background as electric dipoles," Phys. Lett. B 455 (1999) 129-134 [arXiv:hep-th/9901080].

[44] D. Bigatti and L. Susskind, "Magnetic fields, branes and noncommutative geometry," Phys. Rev. D 62 (2000) 066004 [arXiv:hep-th/9908056].

[45] S.-J. Rey, "Exact answers to approximate questions: Noncommutative dipoles, open Wilson lines and UV/IR duality," in: Gravity, Gauge Theories, and Strings, eds. C. Bachas, A. Bilal, M.R. Douglas, N.A. Nekrasov and F. David (Springer, 2002), pp. 587-609 [arXiv:hepth/0207108].

[46] E. Langmann and R.J. Szabo, "Duality in scalar field theory on noncommutative phase spaces," Phys. Lett. B 533 (2002) 168-177 [arXiv:hep-th/0202039].

[47] H. Grosse and R. Wulkenhaar, "Renormalisation of $\phi^{4}$ theory on noncommutative $\mathbb{R}^{2}$ in the matrix base," J. High Energy Phys. 0312 (2003) 019 [arXiv:hep-th/0307017]; "Renormalization of $\phi^{4}$-theory on noncommutative $\mathbb{R}^{4}$ in the matrix base," Commun. Math. Phys. 256 (2005) 305-374 [arXiv:hep-th/0401128].

[48] E. Langmann, R.J. Szabo and K. Zarembo, "Exact solution of noncommutative field theory in background magnetic fields," Phys. Lett. B 569 (2003) 95-101 [arXiv:hep-th/0303082]; "Exact solution of quantum field theory on noncommutative phase spaces," J. High Energy Phys. 0401 (2004) 017 [arXiv:hep-th/0308043]. 
[49] H. Grosse and R. Wulkenhaar, "The beta-function in duality covariant noncommutative $\phi^{4}$ theory," Eur. Phys. J. C 35 (2004) 277-282 [arXiv:hep-th/0402093].

[50] M. Disertori, R. Gurau, J. Magnen and V. Rivasseau, "Vanishing of beta-function of noncommutative $\phi_{4}^{4}$-theory to all orders," Phys. Lett. B 649 (2007) 95-102 [arXiv:hep-th/0612251].

[51] A. Fischer and R.J. Szabo, "Duality covariant quantum field theory on noncommutative Minkowski space," J. High Energy Phys. 0902 (2009) 031 [arXiv:0810.1195 [hep-th]].

[52] H. Grosse and M. Wohlgenannt, "On $\kappa$-deformation and UV/IR mixing," Nucl. Phys. B 748 (2006) 473-484 [arXiv:hep-th/0507030].

[53] P. Bieliavsky, R. Gurau and V. Rivasseau, "Noncommutative field theory on rank one symmetric spaces," arXiv:0806.4255 [hep-th].

[54] F. Lizzi, R.J. Szabo and A. Zampini, "Geometry of the gauge algebra in noncommutative Yang-Mills theory," J. High Energy Phys. 0108 (2001) 032 [arXiv:hep-th/0107115].

[55] A. Armoni, "Comments on perturbative dynamics of noncommutative Yang-Mills theory," Nucl. Phys. B 593 (2001) 229-242 [arXiv:hep-th/0005208].

[56] M. Hayakawa, "Perturbative analysis on infrared aspects of noncommutative QED on $\mathbb{R}^{4}$," Phys. Lett. B 478 (2000) 394-400 [arXiv:hep-th/9912094].

[57] M. Alishahiha, Y. Oz and M.M. Sheikh-Jabbari, "Supergravity and large $N$ noncommutative field theories," J. High Energy Phys. 9911 (1999) 007 [arXiv:hep-th/9909215].

[58] W. Bietenholz, F. Hofheinz and J. Nishimura, "A non-perturbative study of gauge theory on a noncommutative plane," J. High Energy Phys. 0209 (2002) 009 [arXiv:hep-th/0203151].

[59] A. Matusis, L. Susskind and N. Toumbas, "The IR/UV connection in the noncommutative gauge theories," J. High Energy Phys. 0012 (2000) 002 [arXiv:hep-th/0002075].

[60] X. Calmet, B. Jurco, P. Schupp, J. Wess and M. Wohlgenannt, "The standard model on noncommutative spacetime," Eur. Phys. J. C 23 (2002) 363-376 [arXiv:hep-th/0111115].

[61] D.J. Gross, A. Hashimoto and N. Itzhaki, "Observables of noncommutative gauge theories," Adv. Theor. Math. Phys. 4 (2000) 893-928 [arXiv:hep-th/0008075].

[62] E. Langmann and R.J. Szabo, "Teleparallel gravity and dimensional reductions of noncommutative gauge theory," Phys. Rev. D 64 (2001) 104019 [arXiv:hep-th/0105094].

[63] M. Chaichian, M. Oksanen, A. Tureanu and G. Zet, "Gauging the twisted Poincaré symmetry as noncommutative theory of gravitation," Phys. Rev. D 79 (2009) 044016 [arXiv:0807.0733 [hep-th]].

[64] S. Marculescu and F. Ruiz Ruiz, "Seiberg-Witten maps for $S O(1,3)$ gauge invariance and deformations of gravity," Phys. Rev. D 79 (2009) 025004 [arXiv:0808.2066 [hep-th]].

[65] A.H. Chamseddine, " $S L(2, \mathbb{C})$ gravity with complex vierbein and its noncommutative extension," Phys. Rev. D 69 (2004) 024015 [arXiv:hep-th/0309166].

[66] P. Aschieri and L. Castellani, "Noncommutative supergravity in $D=3$ and $D=4$," J. High Energy Phys. 0906 (2009) 087 [arXiv:0902.3823 [hep-th]].

[67] J. Madore, S. Schraml, P. Schupp and J. Wess, "Gauge theory on noncommutative spaces," Eur. Phys. J. C 16 (2000) 161-167 [arXiv:hep-th/0001203]. 
[68] H. Aoki, N. Ishibashi, S. Iso, H. Kawai, Y. Kitazawa and T. Tada, "Noncommutative YangMills in IIB matrix model," Nucl. Phys. B 565 (2000) 176-192 [arXiv:hep-th/9908141].

[69] J. Ambjørn, Y. Makeenko, J. Nishimura and R.J. Szabo, "Finite $N$ matrix models of noncommutative gauge theory," J. High Energy Phys. 9911 (1999) 029 [arXiv:hep-th/9911041]; "Non-perturbative dynamics of noncommutative gauge theory," Phys. Lett. B 480 (2000) 399408 [arXiv:hep-th/0002158]; "Lattice gauge fields and discrete noncommutative Yang-Mills theory," J. High Energy Phys. 0005 (2000) 023 [arXiv:hep-th/0004147].

[70] W. Bietenzholz, A. Bigarini, F. Hofheinz, J. Nishimura, Y. Susaki and J. Volkholz, "Numerical results for $U(1)$ gauge theory on $2 D$ and $4 D$ noncommutative spaces," Fortschr. Phys. 53 (2005) 418-425 [arXiv:hep-th/0501147].

[71] V.O. Rivelles, "Noncommutative field theories and gravity," Phys. Lett. B 558 (2003) 191-196 [arXiv:hep-th/0212262].

[72] H.S. Yang, "Exact Seiberg-Witten map and induced gravity from noncommutativity," Mod. Phys. Lett. A 21 (2006) 2637-2647 [arXiv:hep-th/0402002]; "On the correspondence between noncommutative field theory and gravity," Mod. Phys. Lett. A 22 (2007) 1119-1132 [arXiv:hep-th/0612231].

[73] H. Steinacker, "Emergent gravity from noncommutative gauge theory," J. High Energy Phys. 0712 (2007) 049 [arXiv:0708.2426 [hep-th]]; "Emergent gravity and noncommutative branes from Yang-Mills matrix models," Nucl. Phys. B 810 (2009) 1-39 [arXiv:0806.2032 [hep-th]].

[74] H. Grosse, H. Steinacker and M. Wohlgenannt, "Emergent gravity, matrix models and UV/IR mixing," J. High Energy Phys. 0804 (2008) 023 [arXiv:0802.0973 [hep-th]].

[75] M. van Raamsdonk, "The meaning of infrared singularities in noncommutative gauge theories," J. High Energy Phys. 0111 (2001) 006 [arXiv:hep-th/0110093].

[76] A. Armoni and E. Lopez, "UV/IR mixing via closed strings and tachyonic instabilities," Nucl. Phys. B 632 (2002) 240-256 [arXiv:hep-th/0110113].

[77] S.R. Das and S.-J. Rey, "Open Wilson lines in noncommutative gauge theory and tomography of holographic dual supergravity," Nucl. Phys. B 590 (2000) 453-470 [arXiv:hep-th/0008042].

[78] H. Liu, “`-Trek II: $\star_{n}$ operations, open Wilson lines and the Seiberg-Witten map," Nucl. Phys. B 614 (2001) 305-329 [arXiv:hep-th/0011125].

[79] S.R. Das and S.P. Trivedi, "Supergravity couplings to noncommutative branes, open Wilson lines and generalized star products," J. High Energy Phys. 0102 (2001) 046 [arXiv:hepth/0011131].

[80] A. Dhar and Y. Kitazawa, "Noncommutative gauge theory, open Wilson lines and closed strings," J. High Energy Phys. 0108 (2001) 044 [arXiv:hep-th/0106217].

[81] A. Armoni, E. Lopez and A.M. Uranga, "Closed string tachyons and noncommutative instabilities," J. High Energy Phys. 0302 (2003) 020 [arXiv:hep-th/0301099].

[82] S. Sarkar and B. Sathiapalan, "Aspects of open-closed duality in a background B-field," J. High Energy Phys. 0505 (2005) 062 [arXiv:hep-th/0503009].

[83] N. Ishibashi, S. Iso, H. Kawai and Y. Kitazawa, "String scale in noncommutative Yang-Mills," Nucl. Phys. B 583 (2000) 159-181 [arXiv:hep-th/0004038]. 
[84] Y. Kitazawa and S. Nagaoka, "Graviton propagators in supergravity and noncommutative gauge theory," Phys. Rev. D 75 (2007) 046007 [arXiv:hep-th/0611056].

[85] M. Chaichian, P.P. Kulish, K. Nishijima and A. Tureanu, "On a Lorentz-invariant interpretation of noncommutative spacetime and its implications on noncommutative QFT," Phys. Lett. B 604 (2004) 98-102 [arXiv:hep-th/0408069].

[86] M. Chaichian, P. Presnajder and A. Tureanu, "New concept of relativistic invariance in NC spacetime: Twisted Poincaré symmetry and its implications," Phys. Rev. Lett. 94 (2005) 151602 [arXiv:hep-th/0409096].

[87] H. Grosse and G. Lechner, "Wedge-local quantum fields and noncommutative Minkowski space," J. High Energy Phys. 0711 (2007) 012 [arXiv:0706.3992 [hep-th]]; "Noncommutative deformations of Wightman quantum field theories," J. High Energy Phys. 0809 (2008) 131 [arXiv:0808.3459 [math-ph]].

[88] E. Akofor, A.P. Balachandran and A. Joseph, "Quantum fields on the Grönewold-Moyal plane," Int. J. Mod. Phys. A 23 (2008) 1637-1677 [arXiv:0803.4351 [hep-th]].

[89] S.M. Carroll, J.A. Harvey, V.A. Kostelecky, C.D. Lane and T. Okamoto, "Noncommutative field theory and Lorentz violation," Phys. Rev. Lett. 87 (2001) 141601 [arXiv:hep-th/0105082].

[90] M. Buric, D. Latas, V. Radovanovic and J. Trampetic, "Nonzero $Z \rightarrow \gamma \gamma$ decays in the renormalizable gauge sector of the noncommutative standard model," Phys. Rev. D 75 (2007) 097701 [arXiv:hep-ph/0611299].

[91] A.P. Balachandran and S.G. Jo, " $Z^{0} \rightarrow 2 \gamma$ and the twisted coproduct of the Poincaré group," Int. J. Mod. Phys. A 22 (2007) 6133-6146 [arXiv:0704.0921 [hep-th]].

[92] C. Tamarit and J. Trampetic, "Noncommutative fermions and quarkonia decays," Phys. Rev. D 79 (2009) 025020 [arXiv:0812.1731 [hep-th]].

[93] M. Chaichian, M.M. Sheikh-Jabbari and A. Tureanu, "Hydrogen atom spectrum and the Lamb shift in noncommutative QED," Phys. Rev. Lett. 86 (2001) 2716-2719 [arXiv:hep-th/0010175].

[94] G. Amelino-Camelia, G. Mandanici and K. Yoshida, "On the IR/UV mixing and experimental limits on the parameters of canonical noncommutative spacetimes," J. High Energy Phys. 0401 (2004) 037 [arXiv:hep-th/0209254].

[95] R.C. Helling and J. You, "Macroscopic screening of Coulomb potentials from UV/IR mixing," J. High Energy Phys. 0806 (2008) 067 [arXiv:0707.1885 [hep-th]].

[96] E. Akofor, A.P. Balachandran, A. Joseph, L. Pekowsky and B.A. Qureshi, "Constraints from CMB on spacetime noncommutativity and causality violation," Phys. Rev. D 79 (2009) 063004 [arXiv:0806.2458 [astro-ph]]. 